\title{
ASPECTOS INCOMUNS DA PARACOCCIDIOIDOMICOSE INFANTIL
}

\section{Achiléa Lisboa Bittencourt, Jacy Amaral Freire de Andrade, Edgard Marceiino Carvalho, Fernando Sergio da Silva Badaró e Jair Santos Miranda}

\begin{abstract}
São relatados dois casos de paracoccidioidomicose (PCM) infantil. Em um deles, a principal manifestação da doença foi tumoração abdominal, simulando linfoma maligno não Hodgkin. $O$ outro apresentou acentuado envolvimento articular, com muptura da cápsula. Representam o segundo e terceiro casos de PCM infantil observados na Bahia, ambos procedentes de areas urbanas.
\end{abstract}

Palavras chaves: Paracoccidioidomicose. Blastomicose sul-americana. Paracoccidioidomicose infantil.

É muito pequena a freqüência da PCM em crianças e adolescentes. ${ }^{1} 678$ Dentre 1899 casos, Castro \& Del Negro ${ }^{3}$ encontraram apenas $3,68 \%$ com idade inferior a 15 anos. Recentemente, Londero e cols ${ }^{6}$ fizeram revisão de 45 casos de literatura e apresentaram mais quatro na faixa etária de 9-17 anos. Segundo estes autores, a quase totalidade dos casos infanto-juvenis de PCM do Brasil é proveniente dos estados de São Paulo, Minas Gerais, Rio de Janeiro e Goiás.

A finalidade desta publicação é apresentar o segundo e terceiro casos de PCM infantil observados na Bahia, os quais apresentam aspectos incomuns.

\section{RELATO DOS CASOS}

Caso I - Criança do sexo feminino, com 11 anos, procedente de S. Sebastião do Passé (Bahia) onde reside em zona urbana, com história de 2 meses de emagrecimento, palidez e de tumoração abdominal. Ao exame físico, constataram-se linfadenopatia superficial generalizada, tumoração fixa no mesogástrio com $10 \times 8 \mathrm{~cm}$ e, na face, lesão tuberosa e eritematosa com 0,6x0,4 cm (Fig. 1). A radiografia do tórax revelou linfadenopatia hilar. As suspeitas clínicas foram linfoma maligno não Hodgkin e tuberculose.

Caso II - Criança do sexo masculino, com 12 anos, natural de Simões Filho (Bahia), onde mora em zona urbana. Tinha história de duas semanas de acentuado aumento de volume da coxa e da região da articulação coxo-femural direitas. Ao exame físico, constaram-se acentuado edema com flogose, destas regiōes (Fig. 2) e linfadenopatia cervical e inguinal. À

Pedidos de separatas para Dra. Achiléa Lisboa Bittencourt. Serviço de Anatomia Patológica, Hospital Martagão Gesteira, Rua José Duarte 114, Tororó 40000 Salvador, Bahia. Hospital Martagão Gesteira. Laboratórios de Doenças Infecciosas e Parasitárias, de Anatomia Patológica e de Imunologia do Hospital Prof. Edgard Santos, Universidade Federal da Bahia.

Recebido para publicação em 9/3/87

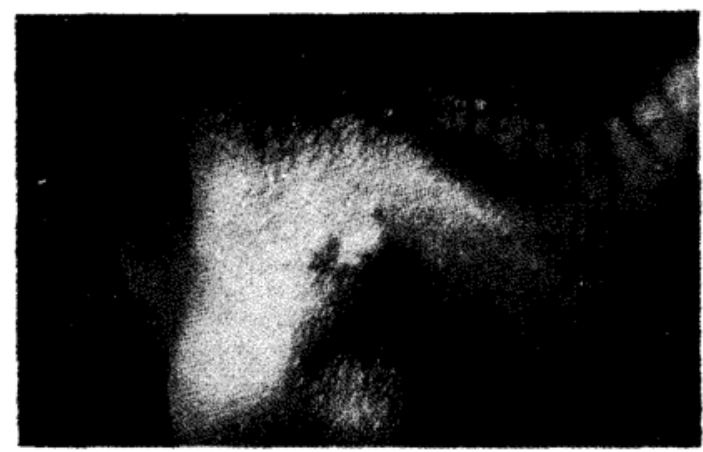

Fig. 1 - Caso I. Lesão tuberosa na face.

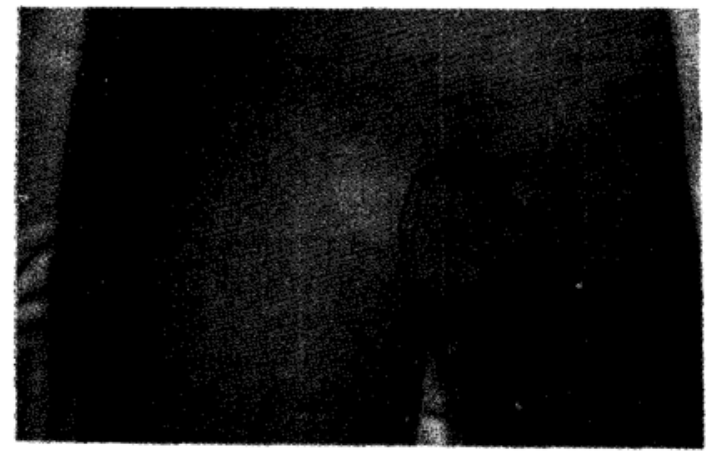

Fig. 2 - Caso II. Observar o acentuado edema da coxa direita.

cirurgia, observou-se ruptura da cápsula da articulação coxo-femural com presença de grande quantidade de material de aspecto purulento, que foi drenado. No ato cirúrgico, foi dado o diagnóstico de artrite piogênica. Estudo radiológico do tórax e dos ossos da bacia e coxa foi normal. Estudo laboratorial de ambos os casos - Sorologia pela técnica da imunodifusão dupla com antigeno de Paracoccidioides brasiliensis foi positiva. Exame direto e cultura foram também positivos para este fungo. Testes cutâneos com a paracoccidioidina, PPD, candidina e tricofitina foram negativos. Foi feita determinação de linfócitos T e B através de técnicas previamente descritas ${ }^{9}$, tendo-se 
Bittencourt AL, Andrade JAF de, Carvalho EM, Badaró FSS, Miranda JS. Aspectos incomuns da paracoccidioidomicose infantil. Revista da Sociedade Brasileira de Medicina Tropical 20: 223-224, Out-Dez, 1987

obtido resultados normais. Avaliou-se também a subpopulação de linfócitos $T$, através do emprego de anticorpos monoclonais anti-OKT 4 e anti-OKT8, não se tendo evidenciado alterações na proporção entre células $T$ auxiliares $\mathrm{e} T$ supressoras. Exame anátomopatológico de linfonodos cervicais, em ambos os casos, mostrou processo granulomatoso com extensa necrose de caseificação e numerosas formas leveduriformes com múltiplo brotamento (técnica de Grocott). No caso I, a biópsia da lesão de pele revelou atrofia epidérmica e reação granulomatosa com numerosas formas de $P$. brasiliensis. Evolução e tratamento-Usouse, em ambos os casos, uma associação de Trimetoprim (320mg/dia) com Sulfametoxazol (1,6g/dia). No caso I, constatou-se desaparecimento das lesões ao final de três meses mas houve posterior descontinuidade do tratamento com recidiva. $O$ outro caso completou agora um ano de tratamento e não apresenta alterações há 10 meses.

\section{COMENTÁRIOS}

Na criança e no adolescente há predominante envolvimento dos órgãos hematopoiéticos. ${ }^{3}$ Londero e cols $^{6}$ classificam a PCM infanto-juvenil de acordo com a predominância do envolvimento e, por ordem de frequiência, em formas linfática superficial, óssea, gastrointestinal, septicêmica, cutânea e respiratória, estas duas últimas de rara ocorrência.

No primeiro caso apresentado, constatou-se extenso envolvimento de linfonodos superficiais e profundos, mais acentuado ao nível dos linfonodos mesentéricos, os quais formavam uma massa tumoral, simulando clinicamente linfoma maligno não Hodgkin e tuberculose. Esta manifestação tem sido descrita pouco freqüentemente na literatura. ${ }^{2} 7$ Além disto, observou-se discreto envolvimento cutâneo, manifestado sob forma de lesão única tuberosa ao nível da face. No outro caso, o envolvimento foi predominantemente articular, com ruptura da cápsula e acentuado edema da pele, na ausência de lesōes ósseas, aspecto este descrito raramente na literatura. ${ }^{7}$

A ausência de reatividade aos testes cutâneos feitos com diversos antígenos indica diminuição da resposta imune mediada por células, fato já observado em outros casos. ${ }^{4}$ É impossivel, contudo, determinar se esta alteração existia ou se foi secundária à infecção micótica. Pode-se, contudo, nestes casos, determinar que a alteração da resposta imune não foi devido à redução de linfócitos $\mathrm{T}$, nem tampouco à modificação na proporção entre células $T$ auxiliares e supressoras no sangue.
Os pacientes com PCM são, em geral, provenientes de zona rural. ${ }^{5}$ No entanto, estes e o outro caso anteriormente observado na Bahia, constituem exemplos desta micose incidindo em indivíduos de zona urbana. $^{2}$

\section{SUMMARY}

The authors present two cases of infantil paracoccidioidomycosis. In one case the main presentation was an abdominal mass that mimicked nonHodgkin lymphoma. In the other case the child had a joint swelling with rupture of the capsule and the clinical diagnosis was piogenic arthritis. They represent the second and third cases of infantile paracoccidioidomycosis observed in the state of Bahia, all coming from urban areas.

Key words: Paracoccidioidomycosis. South American Blastomycosis. Infantile paracoccidioidomycosis.

\section{REFERÊNCIAS BIBLIOGRÁFICAS}

1. Azevedo JF, Lisboa CG. Paracoccidioidomicose: estudo de 160 casos. Jornal de Pneumologia 6: 30-33, 1980.

2. Bittencourt AL, Andrade JAF, Cendon Filha SP. Paracoccidioidomycosis in a four-year-old boy. Mycopathologia 93: 55-59, 1986.

3. Castro RM, Del Negro G. Particularidades clínicas da paracoccidioidomicose na criança. Revista Hospital das Clínicas Faculdade de Medicina de São Paulo 31: 194 198, 1976.

4. Farhat CK, Raimundo MEC, Gaspar JM, Oliveira RB, Brito JF, Ureña IG. Paracoccidioidomicose na criança. Relato de dois casos. Jornal de Pediatria 60: 226-231, 1986.

5. Lacaz CS, Porto E, Martins JEC. Paracoccidioidomicose. Micologia Médica. Fungos, actinomicetos e algas de interesse médico. Sarvier Editora, São Paulo, 1984.

6. Londero AT, Gonçalves AJR, Cruz ML, Rozembaum R, Cunha RQ, Machado ES, Vieira ARM, Carvalho FG, Braga MP, Azevedo ECL, Wanke B, Cruz MFF, Menezes JA. Paracoccidioidomicose disseminada "infanto-juvenil" em adolescentes. Relato de quatro casos e revisão da literatura. Arquivos Brasileiros de Medicina 61: 5-12, 1987.

7. Londero AT, Melo IS. Paracoccidioidomycosis in childhood. A critical review. Mycopathologia 82: 49-55, 1983.

8. Rodriguez C, Piñate FM. La paracoccidioidosis brasiliensis en Venezuela. Estudio de 120 casos. Observaciones clinicas. Gaceta Medica 74: 101-139, 1966.

9. WHO/Iarc Workshop. Special technical report. Identification enumeration and isolation of $B$ and $T$ lymphocytes from human peripheral blood. Scandinavian Jornal of Immunology 3: 521-532, 1974. 